\title{
The Effect of the Sequence of Image Distortion Correction on the Accuracy of Connection Point Generation
}

\section{Yuanyuan Wang*}

Land Surveying, Planning and Design Institute of Shaanxi Provincial Land Engineering Construction Group, Xi'an, 710075, China

Corresponding author: byg503@sina.com

\begin{abstract}
In this paper, the UAV photogrammetry data of two hills and two flat sampling areas are selected. The image data are processed by pixel grid software through different operation methods, and the effect of the two methods is compared. The results show that after the distortion correction, the image is rotated manually or the flight angle in the POS data is directly changed, which has no obvious effect on the error in the number of iterations of the automatic connection point. When the distortion correction is not corrected, the photo is rotated, and the angle of the Omega, Phi and Kappa angles is obtained from the UAV autopilot system, and makes the corner element higher precision when the automatic connection point is generated. Add Kappa to the POS data by 90, and then correct distortion while choosing to rotate pictures; Set the Omega, Phi is 0, Kappa angle from the IMU, this method avoids the influence of the angle deviation caused by the tilt or jitter of the flight process, so it is better to line elements when the automatic connection point is generated.
\end{abstract}

Key words: UAV photogrammetry; pixel grid software; distortion correction; automatic connection point

\section{Introduction}

Due to its advantages such as short operating cycle, less expense and less field workload, and easy access to real-time image data, low-altitude UAV photogrammetry has been widely used in small-scale land use survey, territorial survey and environmental monitoring in recent years. However, in consideration of problems such as carrying a non-measurement camera, short flight baseline and large rotation angle in photogrammetry of low-altitude unmanned aerial vehicle (UAV), the acquired image has various errors inevitably. These errors are generally reduced through software, algorithm and other technical means in data processing. Non-measurement cameras exist optical distortion aberration. The method of distortion correction has changed from the simple linear transformation algorithm in the early stage to the expression of radial and tangential distortion and the subsequent self-calibration. In view of UAV airborne triangulation error, a number of scholars have verified the feasibility of the GPS-assisted bundle block adjustment, and continuously optimized various adjustment processes using algorithms or models. The study of UAV error mainly focuses on the distortion correction and block adjustment, etc. However, there is little research on the error of automatic connection point generation in the early

\footnotetext{
Copyright (C) 2020 by author(s) and Frontier Scientific Research Publishing Inc.

This work is licensed under the Creative Commons Attribution International License (CC BY 4.0).

http://creativecommons.org/licenses/by/4.0/
} 
processing of UAV. In this paper, pixel grid is used as the platform software to process two types of image data of hillock and flat ground through different operation methods, and compare the effects of the two methods on automatic point connection generation results.

\section{Overview of Research Area and Aerial Photography of UAV}

Data of two hill samples (study area 1 and study area 2) came from Xiangmiao Town, Binxian County, Shaanxi province, China, with an average elevation of 1600 meters and a maximum elevation difference of 25.4 meters. Data of two plain samples (study area 3 and study area 4) came from Longting Town, Dali county, Shaanxi province, China, with an average elevation of 940 meters and a maximum elevation difference of 5 meters.

Both air flights used the CK-GY04 UAV, and Canon 5 d Mark II digital cameras used in photographic imaging. The mapping route is uniformly arranged along each research area. The research area 1 and 2 belong to the same flight sortie, and the research area 3 and 4 belong to another flight sortie. The parameters of the specific research area are as follows:

Table 1. Parameters of the study area

\begin{tabular}{|c|c|c|c|c|c|}
\hline Study area & Terrain & $\begin{array}{c}\text { Numbe } \\
\text { r of } \\
\text { routes }\end{array}$ & $\begin{array}{c}\text { Number } \\
\text { of } \\
\text { pictures }\end{array}$ & $\begin{array}{c}\text { Flight } \\
\text { direction }\end{array}$ & Inner orientation element \\
\hline $\begin{array}{c}\text { Study area } \\
1\end{array}$ & Hills & 4 & 36 & North-South & fo $=35.0000 \mathrm{~mm}, \mathrm{Xo}=18.1429 \mathrm{~mm}, \mathrm{Yo}_{\mathrm{o}}=12.4892 \mathrm{~mm}$ \\
\hline $\begin{array}{c}\text { Study area } \\
2\end{array}$ & Hills & 3 & 21 & North-South & $\mathrm{fo}=35.0000 \mathrm{~mm}, \mathrm{Xo}=18.1429 \mathrm{~mm}, \mathrm{Yo}=12.4893 \mathrm{~mm}$ \\
\hline $\begin{array}{c}\text { Study area } \\
3\end{array}$ & Flat & 4 & 44 & North-South & $\mathrm{fo}=34.4846 \mathrm{~mm}, \mathrm{Xo}=17.9268 \mathrm{~mm}, \mathrm{Yo}=11.6769 \mathrm{~mm}$ \\
\hline $\begin{array}{c}\text { Study area } \\
4\end{array}$ & Flat & 4 & 48 & North-South & $\mathrm{fo}=34.4846 \mathrm{~mm}, \mathrm{Xo}=17.9268 \mathrm{~mm}, \mathrm{Yo}=11.6769 \mathrm{~mm}$ \\
\hline
\end{tabular}

\section{Technical Route}

Pixel grid software uses POS system (position and orientation system, airborne high precision positioning attitude system) data to obtain image orientation parameters. Based on feature points and pyramid image matching technology, the corresponding relations of the homologous points on different images are established through relative orientation and model connection.

\subsection{Image distortion correction}

Establish a mathematical model to reduce the distortion caused by camera objective lens or projection center migration, and restore the theoretical position of image points.

\subsection{Interior orientation of digital image}

According to the frame coordinates of the image and the camera calibration parameters, restore the relevant position of the image and the camera. Then determine the conversion relationship between the scanning coordinate system and the frame coordinate system. And correct the image point to the frame coordinate system through the scanning deformation correction.

\subsection{Pyramid image matching}

Adopting the integral image matching method, taking the regular grid as the matching node and the surrounding connection points of the grid as the neighborhood system, the relaxation iteration formula is established to achieve the overall optimal matching through image multilevel matching. 


\subsection{Automatic extraction of aerial triangulation connection point}

The POS system onboard the UAV can obtain the spatial position information and attitude information of flight moments. According to the spatial position information and attitude information, automatic extraction of aerial triangulation connection points is completed through two steps: automatic point selection and automatic relative orientation. Based on the feature point extraction algorithm, automatic point selection refers to selecting points with uniform distribution and obvious features in the overlapping area from multiple overlapping images. The relative orientation is defined as follows: according to the principle of intersection of the corresponding image rays, the relative orientation element values of the two images are solved, and the parameters of the relative position and attitude relationship of the image are determined, thereby establishing a ground stereo model. According to the stereo model after the relative orientation calculation results, the automatically selected feature points are eliminated with gross errors and recalculated until there are no gross errors.

\section{Research Approach}

In this study, the initial exterior orientation elements of the aerial photograph were obtained, the automatic connection points were processed by different methods, and the corresponding exterior orientation elements were obtained again and analyzed after processing. The exterior orientation elements consist of three linear elements and three angular elements. The three linear elements refer to the coordinates $(\mathrm{X}, \mathrm{Y}, \mathrm{Z})$ of the photography center in the ground photo gram-metric coordinate system at the instant of photography. The three angular elements are the spatial attitude parameters (Omega, Phi and Kappa) of the photo in the instant of photography, which respectively represent the lateral inclination angle, heading inclination angle and photo rotation angle. The main treatment methods are as follows:

Processing method 1: Do not rotate the photo when the distortion is corrected. Manually rotate the photo after correction (turn all the images from north to south by $180^{\circ}$ manually), new construction GNSS/IMU - APPOX. Kappa EO setting angle of 0 , finally to return to the interface of step to select images by selecting initial exterior orientation elements using GPS/IMU: X, Y, Z from GPS, Omega, Phi, Kappa angle are obtained from IMU.

Processing method 2: Kappa angle unified POS data plus 90 degrees. Save the original format, aberration correction in the process of rotating photos. The original kappa angle was near $0^{\circ}$, the original photo was flying from south to north, then set it near $90^{\circ}$ and fly from west to east, rotate clockwise. The original kappa angle is around $180^{\circ}$. The original photo was from north to north and south, set it around $270^{\circ}$ and fly from east to west and rotate counterclockwise. Import the modified POS data in GNSS/ IMU-APPOX.EO during the new project. In the image interface, select images by selecting initial exterior orientation elements using GPS/IMU: X, Y and Z were obtained from GPS, Omega and Phi were 0, and Kappa angle was obtained from airstrip direction angle.

\section{Results Analysis and Discussion}

5.1 The influence of different processing methods on the iterative adjustment results of the automatic connection

Compare the results of treatment methods 1 and 2: the number of connection points generated in the same study area was close, but with randomness; the number of adjustment iterations in the same research area and the error in the iteration are the same. In the same research area, $\mathrm{X}$ root mean square error and $\mathrm{Y}$ root mean square error are interchanged numerically due to the change of image direction. To sum up, no matter manual rotation of the image after distortion correction or direct change of the flight Angle in POS data has no significant influence on the iterative adjustment results of automatic connection points. 
Table 2. The influence of different processing methods on the iterative adjustment results of the automatic connection points

\begin{tabular}{|c|c|c|c|c|c|c|}
\hline $\begin{array}{l}\text { Study } \\
\text { area }\end{array}$ & $\begin{array}{c}\text { Processing } \\
\text { method }\end{array}$ & $\begin{array}{c}\text { Connection } \\
\text { points }\end{array}$ & $\begin{array}{c}\text { Adjustment } \\
\text { number of } \\
\text { iterations }\end{array}$ & $\begin{array}{l}\text { Iteration } \\
\text { error } / \mu \mathrm{m}\end{array}$ & X RMSE/ $\mu \mathrm{m}$ & Y RMSE/ $\mu \mathrm{m}$ \\
\hline \multirow{2}{*}{$\begin{array}{l}\text { Study } \\
\text { area } 1\end{array}$} & Method 1 & 23524 & 17 & 4.2 & 3.4 & 3.6 \\
\hline & Method 2 & 23584 & 17 & 4.2 & 3.6 & 3.4 \\
\hline \multirow{2}{*}{$\begin{array}{l}\text { Study } \\
\text { area } 2\end{array}$} & Method 1 & 15719 & 17 & 3.6 & 3.2 & 3.1 \\
\hline & Method 2 & 15755 & 17 & 3.6 & 3.1 & 3.2 \\
\hline \multirow{2}{*}{$\begin{array}{l}\text { Study } \\
\text { area } 3\end{array}$} & Method 1 & 30314 & 11 & 2.5 & 2.1 & 2.1 \\
\hline & Method 2 & 30398 & 11 & 2.5 & 2.1 & 2.1 \\
\hline \multirow{2}{*}{$\begin{array}{l}\text { Study } \\
\text { area } 4\end{array}$} & Method 1 & 32194 & 14 & 2.8 & 2.4 & 2.3 \\
\hline & Method 2 & 32129 & 14 & 2.8 & 2.3 & 2.4 \\
\hline
\end{tabular}

5.2 Effects of different processing methods on the external square elements after automatic connection point generation

It can be seen from the table 3 that the average standard deviation and maximum standard deviation of the line elements after the treatment of method 2 are both less than method 1, and the average standard deviation and maximum standard deviation of the line elements in the two plain research areas are both less than the two hill research areas.

Table 3. Standard deviation of line elements after automatically generated connection points

\begin{tabular}{|c|c|c|c|c|c|c|c|}
\hline \multirow{2}{*}{ Study area } & \multirow{2}{*}{$\begin{array}{c}\text { Processing } \\
\text { method }\end{array}$} & \multicolumn{2}{|c|}{$\begin{array}{c}\text { X Direction standard } \\
\text { deviation/m }\end{array}$} & \multicolumn{2}{c|}{$\begin{array}{c}\text { Y Direction standard } \\
\text { deviation/m }\end{array}$} & \multicolumn{2}{|c|}{$\begin{array}{c}\text { D Direction standard } \\
\text { deviation/m }\end{array}$} \\
\cline { 3 - 8 } & SEM & MSD & SEM & MSD & SEM & MSD \\
\hline \multirow{2}{*}{ Study area 1 } & Method 1 & 2.778 & 3.384 & 2.672 & 3.307 & 2.563 & 3.015 \\
\cline { 2 - 8 } & Method 2 & 2.685 & 3.232 & 2.528 & 3.064 & 2.374 & 2.665 \\
\hline \multirow{2}{*}{ Study area 2 } & Method 1 & 3.074 & 3.527 & 2.918 & 3.451 & 2.708 & 2.98 \\
\cline { 2 - 8 } & Method 2 & 3.077 & 3.459 & 2.890 & 3.393 & 2.642 & 2.814 \\
\hline \multirow{2}{*}{ Study area 3 } & Method 1 & 1.455 & 1.778 & 1.440 & 1.813 & 1.385 & 1.666 \\
\cline { 2 - 8 } & Method 2 & 1.407 & 1.679 & 1.385 & 1.722 & 1.289 & 1.475 \\
\hline \multirow{2}{*}{ Study area 4 } & Method 1 & 1.586 & 1.985 & 1.586 & 2.080 & 1.526 & 1.911 \\
\cline { 2 - 8 } & Method 2 & 1.510 & 1.805 & 1.518 & 1.927 & 1.416 & 1.658 \\
\hline
\end{tabular}

SEM: standard deviation of the mean. MSD: maximum standard deviation

It can be seen from the table 4 that the average standard deviation and maximum standard deviation of the angular elements after treatment of method 1 are less than that of method 2, and the average standard deviation and maximum standard deviation of the line elements of the two plain research areas are less than that of the two hill research areas. 
Table 4. Standard deviation of angle elements after automatically generated connection points

\begin{tabular}{|c|c|c|c|c|c|c|c|}
\hline \multirow{2}{*}{ Study area } & \multirow{2}{*}{$\begin{array}{c}\text { Processing } \\
\text { method }\end{array}$} & \multicolumn{2}{|c|}{ Omega Std/(rad*1000) } & \multicolumn{2}{c|}{ phi Std /(rad*1000) } & \multicolumn{2}{|c|}{ kappa Std /(rad*1000) } \\
\cline { 3 - 8 } & SEM & MSD & SEM & MSD & SEM & MSD \\
\hline \multirow{2}{*}{ Study area 1 } & Method 1 & 3.634 & 3.661 & 3.437 & 3.452 & 3.160 & 3.174 \\
\cline { 2 - 8 } & Method 2 & 3.739 & 3.808 & 3.749 & 3.824 & 3.726 & 3.83 \\
\hline \multirow{2}{*}{ Study area 2 } & Method 1 & 4.230 & 4.261 & 4.077 & 4.089 & 3.856 & 3.871 \\
\cline { 2 - 8 } & Method 2 & 4.430 & 4.541 & 4.450 & 4.569 & 4.450 & 4.623 \\
\hline \multirow{2}{*}{ Study area 3 } & Method 1 & 1.834 & 1.841 & 1.804 & 1.809 & 1.603 & 1.607 \\
\cline { 2 - 8 } & Method 2 & 1.962 & 2.003 & 1.965 & 2.006 & 1.952 & 2.029 \\
\hline \multirow{2}{*}{ Study area 4 } & Method 1 & 1.956 & 1.967 & 1.978 & 1.985 & 1.719 & 1.726 \\
\cline { 2 - 8 } & Method 2 & 2.080 & 2.115 & 2.085 & 2.137 & 2.048 & 2.126 \\
\hline
\end{tabular}

\section{Conclusions}

The number of iterations and the iterative adjustment results are consistent under different processing methods in the same study area. The number of images in the two hill research areas is less than the number of the two plain study areas. However, the number of iterations in the hill image group is more than the number of iterations in the plain image group. At the same time, the error in iterations, $\mathrm{X}$ mean square root error and $\mathrm{Y}$ mean square root error of the automatic point connection of the hill image are greater than that of the plain area. The two groups of hill images and the two groups of plain image groups belong to different areas of the same shot. The image quality tends to be the same between the images of the mountain group images or between the plain images. It is indicated that the number of iterations and the post-iteration adjustment results of these automatic connection points are not affected by different processing methods, and are only related to the quantity of images and the quality of images themselves.

Distortion correction when not rotating photos, manual rotation photos after correction (will all fly from north to south picture manually rotated $180^{\circ}$ ), the processing method (method 1) ensures that all image direction after distortion correction on the flight line due north direction, and angle of the three elements are obtained from the UAV autopilot system. The relative accuracy of the initial angle element is guaranteed to the greatest extent. Therefore, after the method 1 is processed, the automatic joint point is generated and the angle elements standard deviation effect is better.

The initial line elements of processing method 1 and processing method 2 are both obtained from GPS. Processing method 2 sets the lateral inclination angle Omega and the heading inclination angle Phi to 0 . This method avoids the influence of angle deviation caused by tilt or jitter during flight. The proportion of linear elements is guaranteed to the greatest extent in subsequent processing. Therefore, the standard deviation of line elements is better after the automatic connection point is generated by method 2 .

\section{Conflicts of Interest}

The author declares no conflicts of interest regarding the publication of this paper.

\section{References}

[1] Tang M. (2014). Research on Several Key Issues in Preprocessing and Matching of Sequence UAV Images. Southwest Jiaotong University.

[2] Ahdel-Aziz Y.I. and Karara H.M. (1971). Direct Linear Trasformation into Object Space Coordinates in Close-T-allge Photogram Etry. In proceedinds Syypisium Close-Range Photogramm Etry. Universiy of Illinois at Urbana-Calibration, urbana, Illinois American, 1-18. 
[3] Binger N. (1987). The Application of Laser Radar Technology. Sensors, 4(4): 42-44.

[4] Ji Q. and Dai S. (2004). Self-calibration of a Rotating Camera with a Translational Offset. Robotics \& Automation IEEE Transactions on, 20(1): 1-14.

[5] Friess P. (1991). Aerotriangulation with GPS-Methods, Experience, Exception. Proceedings of the 43rd Phtogrammetric Week, Stuttgart University, 43-49.

[6] Ackermann F. (1992). Prospects of Kinematic GPS for Aerial Triangulation. International Journal of Applied Earth Observation \& Geoinformation, (4): 326-338.

[7] Li D.R. and Yuan X.X. (1995). GPS-supported Bundle Block Adjustment -- An Empirical Results from Test Field Taiyuan. Acta Geodaetica et Cartographica Sinica, (2): 7.

[8] Zheng M.T., Zhang Y.J. and Zhu J.F. (2017). A Fast and Effective Block Adjustment Method with Big Data. Acta Geidaetica et Carrographica Sinica, 46(2): 188-197.

[9] Liu J., Wang L.M. and Yang L.B. (2015). Geometric Correction of GF-1 Satellite Images Based on Block Adjustment of Rational Polynomial Model. Transactions of the Chinese Society of Agricultural Engineering, 31(22): 146-154.

[10] Yuan X.X. (2001). The Principle and Application of GPS Assisted Aerial Triangulation. Surveying and Mapping Press.

[11] Zhang J.Q. (2003). Photogrammetry. Wuhan University Press.

[12] Lin J.J. and Cang G.H. (2005). Photogrammetry. National Defense Industry Press, Beijing. 